\title{
An Optomechanical Oscillator on a Silicon Chip
}

\author{
Mani Hossein-Zadeh, Member, IEEE, and Kerry J. Vahala, Senior Member, IEEE
}

(Invited Paper)

\begin{abstract}
The recent observation on radiation-pressure-driven self-sustained oscillation in high- $Q$ optical microresonators has created new possibilities for development of photonic devices that benefit from unique functionalities offered by these "optomechanical oscillators" (OMOs). Here, we review the physics, fundamental characteristics, and potential applications of OMOs using the silica microtoroidal OMO as an example.
\end{abstract}

Index Terms-Optical oscillators, optical resonators, optomechanical interaction.

\section{INTRODUCTION}

$\mathbf{T}$ HE COUPLING between electromagnetic radiation and mechanical displacement of a body absorbing or reflecting the radiation has long been of scientific interest. However, since the late 1960s [1], increasing interest in this subject has been triggered by the effect of optical forces on high-sensitivity displacement measurements using interferometers [1]. Early experimental studies were performed using a UHF waveguide resonator coupled to a mechanical resonator [2]. It was shown that the presence of the resonant UHF field introduces additional electromagnetically induced damping/stiffness to the mechanical resonator [2]. Quantum limitations set by radiation pressure to interferometric-based measurement of ultraweak forces were also theoretically identified [3]-[5]. Later, experiments performed at optical frequencies reported optical bistability induced by radiation pressure in a cavity [6]

Over time, interest in optical forces within the context of optical resonators has broadened. The idea of using the optomechanical interaction to study a range of quantum phenomena involving a macroscale mechanical oscillator system (i.e., interferometer mirror as a mechanical mode) is, itself, now a powerful driver of fundamental studies (see [14] and references therein). The realization of many of these quantum phenomena requires ultracold mechanical mirror modes in which thermal motion is suppressed so as to reveal inherent quantum behavior. The so-called ground-state cooling is, as a result, now an objective of numerous research groups. Originally conceived as a means of quieting thermal motion in gravity wave detection [7]

Manuscript received May 4, 2009; revised August 4, 2009. First published November 10, 2009; current version published February 5, 2010. This work was supported in part by the Defense Advanced Research Projects Agency, in part by the Caltech Lee Center for Advanced Networking, and in part by Caltech Center for Physics of Information.

M. Hossein-Zadeh is with the Center for High Technology Material, and the Department of Electrical and Computer Engineering, University of New Mexico, Albuquerque, NM 87106 USA (e-mail: mhz@chtm.unm.edu).

K. J. Vahala is with the Department of Applied Physics, California Institute of Technology, Pasadena, CA 91101 USA (e-mail: vahala@ caltech.edu).

Color versions of one or more of the figures in this paper are available online at http://ieeexplore.iee.org.

Digital Object Identifier 10.1109/JSTQE.2009.2031066 (and even earlier as a basic manifestation of coupled, damped oscillators [8]), a leading technique for ground-state cooling applies intrinsic cavity delay to set up radiation pressure damping and cooling of a mirror mechanical mode. More generally, this radiation-pressure cooling belongs to a class of phenomena associated with dynamical back action [3], and includes parametric instability [9], which is discussed shortly and is central to the current study.

Alongside improved understanding of the basic physics of optomechanical interactions in resonators, a revolution in the fabrication of high- $Q$ micro(nano)optical microcavities and high- $Q$ mechanical resonators has lead to a series of beneficial improvements in properties essential to observation of radiation-pressure dynamical phenomena. High optical-Q, essential for large resonant optical power build-up factors; small form factor, necessary to enhance a key parameter that controls the optomechanical coupling strength; and low-mass vibrational modes have become possible in only the last five years. The net effect of these developments has been the observation of radiation pressure and dynamical back-action phenomena associated with cavity delay [7], [9]. Specifically, optomechanical amplification and cooling of mechanical modes have been systematically reported across a wide range of experimental platforms [10]-[22]. These effects work as follows. When blue-detuned, relative to an optical resonance, a pump laser reduces the effective damping (amplification) of the mechanical resonator (mirror mechanical mode), and eventually, triggers self-sustained optomechanical oscillations [10]-[15]. On the other hand, when it is red detuned, damping of the mechanical mode results that also reduces its thermal motion (cooling) [17]-[22]. Although originally studied theoretically in the context of weak force detection (e.g., gravitational waves) [8], [9], these effects were first observed in microstructures featuring both optical and mechanical resonators [10]-[23]. Among these structures, silica microtoroid optical resonators provided the first observations of optomechanical oscillation [10]-[13], and later were among the first reports to demonstrate cooling [17]. As noted before, optomechanical cooling by this method is one of the most promising approaches to achieve the quantum ground state, and therefore, has been extensively treated in the literature [17]-[22].

Here, we focus on optomechanical oscillation and its potential applications in future photonic systems. Silica microtoroid optomechanical oscillators (OMOs) are the first functional on-chip optomechanical devices reported. In an OMO, the continuous source of pump laser power generates mechanical vibrations at RFs without utilizing any sort of external feedback system. These oscillations imprint onto the transmitted optical power that acts as an optical carrier for the RFs. The unique geometry and optomechanical properties of silica microtoroids enable 
efficient coupling between high- $Q$ optical and mechanical modes through radiation pressure, and leads to self-sustained optomechanical oscillations of the microtoroid structure at relatively low input powers $(<0.5 \mathrm{~mW})$. Preliminary characterization of oscillation frequency and phase noise [23] has confirmed that the microtoroid OMO can function as an optical frequency reference (photonic clock) and is a potential candidate in certain RF photonic systems [24]. Although OMOs in this paper are based on high- $Q$ silica microtoroids driven by radiation pressure, the treatments and outcomes are valid for future OMOs designed based on other geometries and materials. In particular, a range of new structures that employ gradient optical forces in photonic crystal resonators [44] fall within the present analysis, and point toward important new directions of study.

\section{RESONANT OPTOMECHANICAL INTERACTION: PHYSICS AND CONCEPTS}

\section{A. Optomechanical Forces: Radiation Pressure and Bolometric Force}

In the absence of optical nonlinearities, electromagnetic radiation can interact with a dielectric structure by: 1) radiation pressure (including both scattering- and gradient-based forces) or 2) bolometric forces. Of the former, we consider here only the scattering-based forces such as the force exerted on a mirror upon reflection of a beam. Radiation pressure is defined as the radiation force per unit area exerted by electromagnetic radiation. The bolometric force is an indirect force resulting from the heat generated in a structure due to optical absorption. The elevated temperature generates thermal stress, and eventually, deflection. This type of optical force is naturally retarded due to the finite time of thermal conductance. The magnitude and the direction of bolometric force (and, therefore, the resulting deflection) strongly depend upon the geometry and composition of the absorbing object. Efficient optomechanical interaction requires low optical absorption. In this case, the bolometric force can generate measurable effects only when it is magnified using bimorph structures [22], [25]. For most applications, the fast time response and, significantly, its nondissipative nature make radiation pressure superior to bolometric forces.

\section{B. Resonant Optomechanical Interaction}

In the absence of optical resonance, the optically induced force through any of the aforementioned mechanisms is very small. Hence, for typical microstructures, the optical power required to generate measurable mechanical deformation (larger than the amplitude of thermal motion) is relatively large. Moreover, in most configurations, nonresonant interaction (i.e., nonoptical-cavity based) can induce only static effects where the optical force creates a constant deformation proportional to the optical intensity. On the other hand, using optical resonators, the optomechanical interaction can be significantly magnified. Furthermore, in this resonant case, the impact of the mechanical microstructure motion on circulating optical power will also be magnified. Relatively small motions will cause large changes in circulating optical power due to the high cavity Finesse.

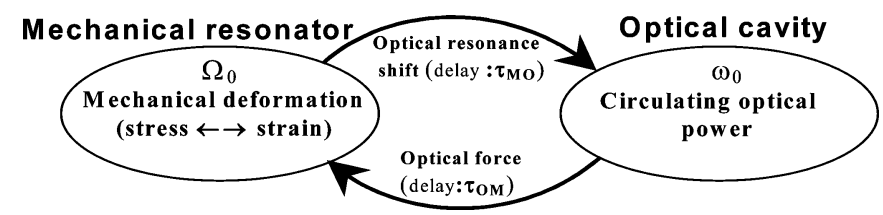

Fig. 1. Dynamic of optomechanical interaction between an optical cavity and a mechanical resonator.

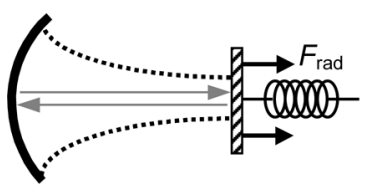

(a)

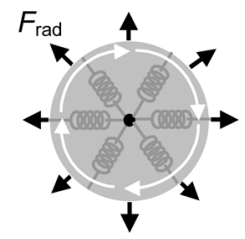

(b)
Fig. 2. (a) FP cavity with one moving flat mirror and a fixed spherical mirror. (b) Circular optical cavity with moving boundary (supporting WG modes).

This overall process is described by a parametric interaction in the Hamiltonian, describing the cavity and mechanical system. Fig. 1 provides a schematic diagram summarizing this interaction between a mechanical resonator and an optical cavity through optomechanical forces.

As indicated in the diagram, the circulating optical power exerts an optomechanical force on the mechanical resonator, and, in response, the mechanical deformation changes the magnitude of the circulating optical power by shifting the optical resonant frequency. The response of the optomechanical force upon change of the mechanical deformation is delayed by $\tau_{\mathrm{OM}}$. For radiation pressure, $\tau_{\mathrm{OM}}$ is the buildup time of the optical cavity $\left(\tau_{\mathrm{L}}=Q_{\mathrm{L}} / \omega_{0}\right.$, where $Q_{\mathrm{L}}$ is the loaded quality factor of the cavity). For bolometric forces, $\tau_{\mathrm{OM}}$ is the sum of $\tau_{\mathrm{L}}$ and the thermomechanical delay (time needed for conversion of optically generated heat to mechanical stress). So far, two most common configurations that enable resonant optomechanical interaction are based on linear and circular (i.e., whispering gallery) geometries. In a Fabry-Perot (FP) optical cavity with a movable mirror, the linear optical path length is modulated by the mirror motion [see Fig. 2(a)]. In a Whispering-Gallery (WG) optical cavity, the circular optical path length is modulated by the radial motion of the cavity [see Fig. 2(b)]. In the absence of loss, the total radiation pressure force $\left(F_{\mathrm{rad}}\right)$ for a linear FP cavity is equal to $2 n P_{c} / c$, and for a circular cavity, it is equal to $2 \pi n P_{c} / c$ (where $c$ is the speed of light, $n$ is the effective refractive index of the optical mode and $P_{c}$ is the circulating optical power inside the cavity).

Note that $F_{\text {rad }}$ is the total force experienced by the reflecting object (integrated over the whole reflecting surface). In the case of the circular cavity, the WG mode is being "reflected" by the interface throughout the whole optical path, while in the FP resonator, the reflection occurs at a single point along the optical path length.

\section{Optomechanical Coupling Factor and Effective Mass}

The sensitivity of the optical resonant frequency $\left(\omega_{0}\right)$ to mechanical deformation defines the strength of the parametric op- 
tomechanical interaction between the corresponding optical and mechanical modes. This sensitivity is quantified by "optomechanical coupling factor" $g_{\mathrm{OM}}=d \omega_{\mathrm{O}} / d \xi$, where $\xi$ is a normal mechanical-mode coordinate of the microstructure that induces the change in the optical path length. Consequently, the optical resonant frequency can be written as $\omega_{\mathrm{O}}(\xi)=\omega_{\mathrm{O}}+g_{\mathrm{OM}} \xi$. If the laser frequency $(\omega)$ is originally detuned from the optical resonance by $\Delta_{0}=\omega-\omega_{0}$, then in the presence of the optomechanical interaction, $\Delta_{0}$ is modified as $\Delta(\xi)=\omega-\omega_{0}(\xi)=$ $\Delta_{0}-g_{\mathrm{OM}} \xi$. Another critical parameter is the effective mass of the mechanical motion $\left(m_{\mathrm{eff}}\right)$. The parameter $m_{\mathrm{eff}}$ may be defined as $2 U / \xi_{m}^{2} \Omega^{2}$, where $U$ is the energy stored in the mechanical mode and $\xi_{m}$ is the amplitude or the maximum value of $\xi$ [23]. $m_{\text {eff }}$ is controlled by the size, material, and the geometry of the optomechanical system. Also, clearly, $m_{\mathrm{eff}}$ changes for different mechanical modes.

\section{Optical and Mechanical Quality Factor}

The energy loss rates in a resonant optomechanical system (coupled optical and mechanical resonators), as characterized by the quality factors of the mechanical resonator and the optical cavity, play differing roles. The strength of the optomechanical interaction for a given optical input power is directly affected by the optical $Q$-factor (since it affects the circulating optical power). The mechanical quality factor, on the other hand, sets a loss rate at which mechanical oscillation is possible by way of optomechanical amplification. The mechanical quality factor is limited by external friction $\left(\gamma_{\text {ext }}\right)$, internal friction $\left(\gamma_{\text {int }}\right)$, and clamping loss $\left(\gamma_{\text {clamp }}\right)$. All these losses depend on the geometry of the mechanical resonator, material properties of the resonant structure, and the deformation of the mechanical mode under test. The mechanical- $Q$ can be expressed as $Q_{\text {mech }}=\Omega_{0} / \gamma_{0}$, where $\Omega_{0}$ is the mechanical resonance frequency and $\gamma_{0}=\gamma_{\text {ext }}+\gamma_{\text {int }}+\gamma_{\text {clamp }}$. The loaded quality factor of the optical cavity $\left(Q_{\mathrm{L}}\right)$ quantifies all optical losses. These include coupling loss, scattering loss, absorption loss, and radiation loss. $Q_{\mathrm{L}}\left(=\omega_{0} \tau_{\mathrm{L}}\right.$, where $\tau_{\mathrm{L}}$ is the total or loaded cavity decay time) is commonly expanded as $1 / Q_{\mathrm{L}}=1 / Q_{0}+$ $1 / Q_{\mathrm{e}}$, where $Q_{\mathrm{e}}\left(=\omega_{0} \tau_{\mathrm{e}}\right.$, where $\tau_{\mathrm{e}}$ is the external cavity decay time) is the quality factor associated with coupling loss, and $Q_{0}\left(=\omega_{0} \tau_{0}\right.$, where $\tau_{0}$ is the intrinsic cavity decay time) is the intrinsic quality factor associated with all other optical loss mechanisms. The main challenge in designing efficient optomechanical systems is finding geometries and material systems that: 1) support high- $Q$ optical and mechanical resonances and 2) enable strong optomechanical coupling (large values of $\left.g_{\mathrm{OM}}\right)$. So far, silica microtoroid resonators [26], [27], suspended silicon waveguide [43], suspended SiN photonic crystal [44], silicon cantilevers [18]-[20], [22], [25], and SiN membranes [16] have been employed in optomechanical systems. Among these, only the silica microtoroid and suspended SiN photonic crystal combine both optical and mechanical resonators in a single microstructure. Other approaches separate these elements as, for example, shown in Fig. 2(a) where the mechanical oscillator is a distinct physical element (mirror) within the FP cavity. In order to obtain high optical- $Q$ and Finesse, the length of the FP cav- ity, as well as the mirror diameter, must be relatively large (to store more energy and avoid diffraction losses). However, this tends to reduce the optomechanical coupling parameter $g_{\mathrm{OM}}$. Typically, a silica microtoroid has a diameter of $50 \mu \mathrm{m}$ while an FP cavity is at least $2 \mathrm{~mm}$ long.

\section{E. Optomechanical Oscillation and Cooling}

In a high- $Q$ resonant optomechanical system, the coupled dynamic of the mechanical displacement and the circulating optical power can be expressed in terms of a modified, effective mechanical dynamic caused by the nonadiabatic response of the optical field to changes in the optical path length, or the so-called "dynamic back-action" [9]. Dynamic back action was considered theoretically in the context of interferometric gravitational wave experiments. In particular, while considering the dynamics of a suspended mirror and the circulating optical power, Braginsky and coworkers predicted that under certain conditions, the optomechanical force (specifically radiation pressure) within an optical resonator can lead to a parametric instability [9] or be used as a tranquilizer for cooling the mechanical modes of the mirror [7]. Although certain static effects of resonant optomechanical interaction have been reported previously [6], the dynamical manifestations of this interaction have only recently become an experimental reality [10]-[25], [28], [29], [31]. This has been enabled by the aforementioned developments in fabrication of high-quality high- $Q$ optical microcavities [26] and mechanical resonators. The first observation of the cooling effect was reported in 2004 in an FP cavity with a micrometer size cantilever mirror using the bolometric force [22]. Two years later (2006), the radiation-pressure-based cooling effect was reported in a cantilever-based FP, as well as using a microtoroidal optical cavity [17]-[21]. The first observation of radiation-pressurebased optomechanical oscillation was reported in 2005 in microtoroid cavities [10]-[12]. Recently, it has been shown that the bolometric force can also give rise to optomechanical oscillation of a cantilever mirror in an FP cavity [25]. So far, silica microtoroidal cavities are the only on-chip-based devices that have been successfully used for efficient optomechanical cooling and oscillation without the need for an external optical cavity.

The dynamics of radiation-pressure-based resonant optomechanical interaction in the classical regime can be explained through coupled equations of motion for resonant optical and mechanical modes [13] as

$$
\begin{aligned}
& \ddot{\xi}(t)+\gamma_{0} \dot{\xi}(t)+\Omega_{0}^{2} \xi(t)=\frac{1}{m_{\mathrm{eff}}}\left[F_{\mathrm{rad}}(t)+F_{\mathrm{L}}(t)\right] \\
& \dot{a}(t)+a(t)\left[\frac{\omega_{0}}{2 Q_{\mathrm{L}}}-i \Delta(\xi)\right]=i \sqrt{\frac{\omega_{0}}{Q_{\mathrm{e}}}|S|^{2}}
\end{aligned}
$$

where " $a$ " is the circulating optical field inside the cavity (with normalization such that $a^{2}$ is the stored energy) and $S^{2}$ is the input optical power $(P) . F_{\mathrm{L}}$ denotes the random Langevin force and ensures that the total steady-state energy in the absence of optomechanical interaction is equal to $k_{B} T$ (fluctuation dissipation theorem). Later, we also introduce this force as an operator to incorporate quantum noise into the analysis. The two 
differential equations are coupled via $\Delta(\xi)\left(=\Delta_{0}-g_{\mathrm{OM}} \xi\right)$ showing the importance of $g_{\mathrm{OM}}$, and also through the radiation force in the (1). Effective parameters for the mechanical motion can be derived by solving the coupled equations of motion using two different perturbative methods [13]: 1) method of retardation expansion [10], [13] and 2) the sideband formalism [13]. The first method is valid in the weak retardation regime when $\Omega \ll 2 \delta\left(2 \delta=1 / \tau_{\mathrm{L}}\right.$ is the bandwidth of the loaded optical resonance), while the second method is also valid in the regime where the mechanical frequency is comparable to or even exceeds the cavity decay rate $(\Omega \gg 2 \delta)$, the so-called resolved sideband regime [21]. Using the second method, assuming that $\xi=\xi_{0} \cos (\Omega t)$, and keeping only the first-order perturbation term for the amplitude of the optical field inside the cavity, the radiation force $\left(F_{\mathrm{rad}}\right)$ can be expressed in terms of in-phase $\left(F_{\mathrm{I}}\right)$ and quadrature $\left(F_{\mathrm{q}}\right)$ components with respect to $\xi(t)$ [13]. The magnitude of these forces are determined by the parameters of the optical cavity (refractive index, radius, coupling factor, laser frequency, $Q_{0}, Q_{\mathrm{ex}}$, and $Q_{\mathrm{L}}$ ), as well as $\Delta_{0}, \Omega_{0}$, and $P$. $F_{\mathrm{I}}$ modifies the mechanical rigidity, and consequently, the resonant frequency of the cavity such that $\Omega=\Omega_{0}+\Delta \Omega_{\mathrm{OM}}$, where $\Delta \Omega_{\mathrm{OM}}$ is the optomechanical resonance shift. This effect is referred to as "optical spring effect" [28], [29]. $F_{\mathrm{q}}$ changes the damping factor such that $\gamma_{\mathrm{eff}}=\gamma_{\mathrm{OM}}+\gamma_{0}$, where $\gamma_{\mathrm{OM}}$ is the modification due to the radiation forcẽ $\gamma_{\mathrm{OM}}$, and $\Delta \Omega_{\mathrm{OM}}$ can be written as [13]

$$
\begin{aligned}
\gamma_{\mathrm{OM}} & =-F^{2} A C\left(\frac{1}{4 \tau_{\mathrm{L}}^{2} B_{-}^{2}+1}-\frac{1}{4 \tau_{\mathrm{L}}^{2} B_{+}^{2}+1}\right) P \\
\Delta \Omega_{\mathrm{OM}} & =F^{2} A C \tau_{\mathrm{L}}\left(\frac{B_{-}}{4 \tau_{\mathrm{L}}^{2} B_{-}^{2}+1}-\frac{B_{+}}{4 \tau_{\mathrm{L}}^{2} B_{+}^{2}+1}\right) P \\
A & =\frac{8 n^{2} \omega_{0}}{\Omega_{0} m_{\mathrm{eff}} c^{2}}, \quad C=\frac{\tau_{\mathrm{L}} / \tau_{\mathrm{e}}}{4 \Delta_{0} \tau_{\mathrm{L}}^{2}+1}, \\
B_{-} & =\Delta_{0}-\Omega_{0}, \quad \text { and } \quad B_{+}=\Delta_{0}+\Omega_{0}
\end{aligned}
$$

where $F$ is the finesse of the optical cavity $\left(F=2 \pi Q_{\mathrm{L}} / T_{\mathrm{rt}} \omega_{0}\right.$ and $T_{\text {rt }}$ is the cavity round-trip time) and $n$ is the effective refractive index of the optical mode circulating in the cavity. The magnitude of $\gamma_{\mathrm{OM}}$ can be adjusted by $P$ and $\Delta_{0}$. The sign of $\gamma_{\mathrm{OM}}$ is equal to $-\operatorname{sgn}\left(\Delta_{0}\right)$ such that if the pump laser is reddetuned $\left(\Delta_{0}<0\right)$, the optomechanical damping factor $\left(\gamma_{\mathrm{OM}}\right)$ is positive and the optomechanical force augments the intrinsic mechanical damping. This damping can also be shown to be accompanied by mechanical-to-optical power flow, a phenomenon called radiation pressure cooling that is currently the subject of intensive research [17]-[22]. As an aside, radiation pressure cooling does not add thermal noise, and calculations show that it can reduce the Brownian noise of the coupled mechanical mode to such a low level that quantum mechanical behavior (normally screened by thermal noise) can be observable [13], [14]. If the pump laser is blue-detuned $\left(\Delta_{0}<0\right)$, the optomechanical damping factor $\left(\gamma_{\mathrm{OM}}\right)$ is negative, which means the optomechanical force amplifies the mechanical motion. In the blue-detuned regime when the optomechanical gain cancels the intrinsic mechanical loss $\gamma_{0}=-\gamma_{\mathrm{OM}}$ (the threshold condition), the effective mechanical loss $\left(\gamma_{\text {eff }}\right)$ becomes zero
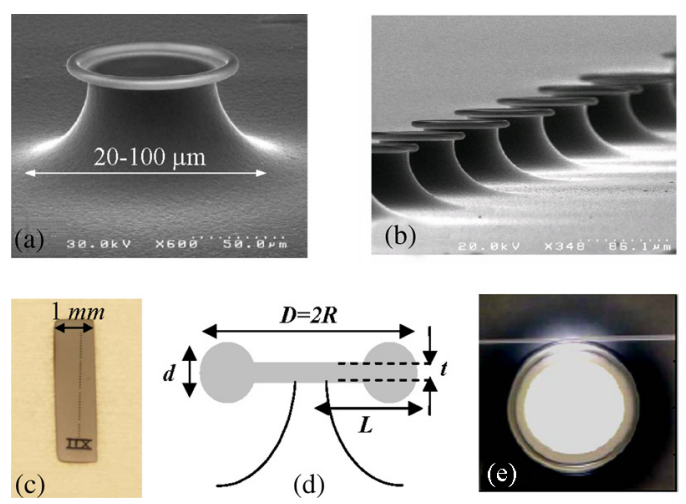

Fig. 3. (a) SEM image of silica microtoroid. (b) SEM image of a series of silica microtoroids fabricated side-by-side. (c) Silicon chip containing a row of 40 microtoroids. (d) Schematic diagram of microtoroid showing the main geometrical parameters. (e) Microphotograph of a silica microtoroid coupled to a silica fiber taper.

and regenerative optomechanical oscillation starts [10]-[13]. For a given optomechanical resonator and laser detuning $\left(\Delta_{0}\right)$, this occurs when the pump power level is above the threshold pump power $P_{\mathrm{th}} . P_{\mathrm{th}}$ can be derived by equating $\gamma_{\mathrm{OM}}$ and $\gamma_{0}$ $\left(=\Omega_{0} / Q_{\text {mech }}\right)$ as $[13]$

$$
P_{\mathrm{th}}=\frac{\Omega_{0}^{2} m_{\mathrm{eff}} c^{2}}{Q_{\mathrm{mech}} 8 \omega_{0} n^{2} F^{2} C}\left(\frac{1}{4 \tau_{\mathrm{L}}^{2} B_{-}^{2}+1}-\frac{1}{4 \tau_{\mathrm{L}}^{2} B_{+}^{2}+1}\right)^{-1} .
$$

Clearly, from inspection of this equation, the benefits of small $m_{\text {eff }}$, large $Q_{\text {mech }}$, and large Finesse are apparent. The role of the Langevin force in the optomechanical oscillation linewidth (above threshold) is also crucial and cannot be ignored (see Section III-C). In the next section, we focus on radiationpressure-driven $\mathrm{OMO}$ and use the silica microtoroid resonator as the platform.

\section{RADIATION-PRESSURE-DRIVEN OMO}

\section{A. Silica Microtoroid OMO}

Ultrahigh- $Q$ (UH- $Q$ ) microtoroidal silica resonators represent a distinct class of optical resonators with $Q$ s in excess of 100 million [27]. This exceptional quality factor is a result of employing a special fabrication process that generates surfacetension-induced smoothness on the resonator sidewalls. Silica microtoroids are fabricated on a silicon wafer through a relatively simple fabrication process that allows fast production of UH- $Q$ microtoroids with high yield. Fig. 3(a) is an electron microscope image of a silica microtoroid resonator.

As shown in Fig. 3(b), large numbers of microtoroids can be fabricated side by side in a row (also see Fig. 3(c), which shows a silicon chip with a row of 40 microtoroids). The geometry of a microtoroid is specified by the major diameter $(D=2 R)$, minor diameter $(d)$, silica membrane thickness $(t)$, and the undercut $(L)$, as shown in Fig. 3(d). For a typical microtoroid, $t \sim 2 \mu \mathrm{m}$, $d \sim 3-10 \mu \mathrm{m}, D \sim 20-80 \mu \mathrm{m}$, and $L \sim 2-40 \mu \mathrm{m}$. The WG optical mode [26] circulates around the circumference of the toroidal section at the silica-air boundary. Coupling optical power into and out of the microtoroid resonator is accomplished by fiber 


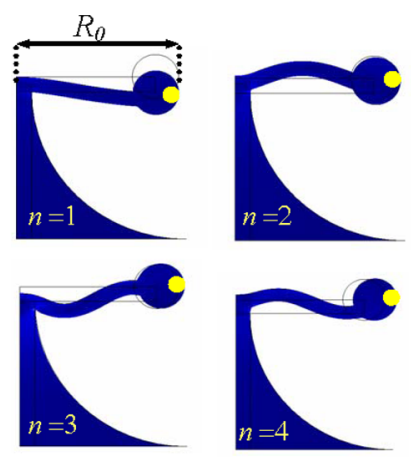

(a)

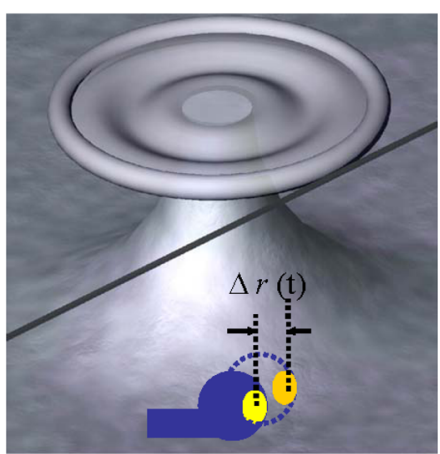

(b)
Fig. 4. (a) First four mechanical eigen modes of a silica microtoroid. (b) 3-D rendering of the fourth mechanical mode of the silica microtoroid.

taper coupling [30] [see Fig. 3(e)]. The silica microtoroid also supports relatively high- $Q$ mechanical modes $\left(Q_{\text {mech }} \sim 10^{3}-\right.$ $10^{4}$ ). Fig. 4(a) shows the first four eigen mechanical modes of a silica microtoroid. Fig. 4(b) is a 3-D rendering of the fourth mode, and the inset shows the radial change $(\Delta r(\mathrm{t}))$ resulting from the corresponding mechanical displacement. For a typical microtoroid, the resonant frequencies of the first five modes are between 5-100 $\mathrm{MHz}$. The mechanical quality factor $\left(Q_{\text {mech }}\right)$ of these modes strongly depends on the geometry (pillar size, $D, d$, and symmetry), as well as the quality of the dry etching. Typically, $Q_{\text {mech }} \sim 500-1000$ for the first mode and $Q_{\text {mech }} \sim$ 2000-5000 for $n=3,4$, and 5 when measured in the air. Many of these mechanical modes feature motion that is strongly coupled to the circular optical path length (an exception is the second-order mode for which there is only weak radial motion). For a microtoroid, $\xi=r, \xi_{0}=R_{0}$, and $g_{\mathrm{OM}}=-\omega_{0} / R_{0}$. These also feature low effective mass $\left(m_{\mathrm{eff}} \sim 10^{-11} \mathrm{~kg}\right.$ for third and fourth-order modes). The combination of high optical and mechanical quality factors, and the low $m_{\text {eff }}$, makes the silica microtoroid a superior platform for exploring various aspects of radiation-pressure-based optomechanical interaction.

In a microtoroidal cavity, the contribution of bolometric forces in the dynamic of OMO is negligible due to its small magnitude, as well as slow response, relative to the RF rates of the mechanical modes. Also, since the resonant optical field is tightly confined inside the dielectric medium (silica) and moves with the dielectric boundary, the optical gradient force is absent.

Fig. 5(a) shows an scanning electron micrograph (SEM) of a silica microtoroid. The diameter of the silicon pillar of this microtoroid has been specifically reduced to lower the threshold for the fourth mechanical mode (i.e., $m_{\mathrm{eff}}$ is reduced and $Q_{\text {mech }}$ is improved). Fig. 5(b) is the top-view photomicrograph of the same microtoroid coupled to a fiber taper. Fig. 5(c) is the schematic diagram of the typical experimental arrangement for characterizing the microtoroid OMO. The OMO shown in Fig. 5(a) has a radius of $R_{0}=30 \mu \mathrm{m}$ and $Q_{0}=5.5 \times 10^{6}$. At critical coupling $\left(Q_{0}=Q_{\mathrm{e}}\right)$, the measured value of the $P_{\mathrm{th}}$ for the fourth mechanical mode of this microtoroid is $250 \mu \mathrm{W}$, which is in good agreement with the calculated value of $222 \mu \mathrm{W}$ (given the uncertainties in the measurement). The oscillating mechanical mode has a frequency of $f_{\text {mech }}=\Omega_{0} / 2 \pi=54.2 \mathrm{MHz}$

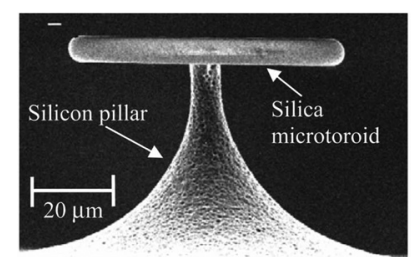

(a)

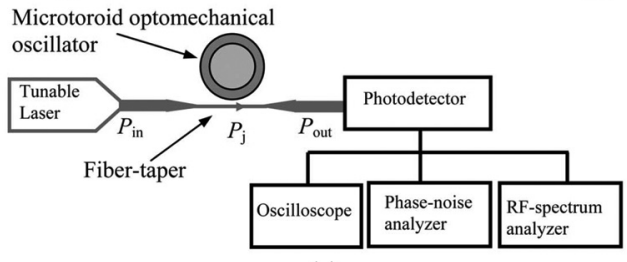

(c)

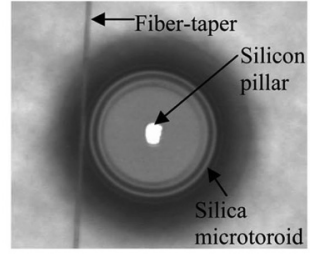

(b)
Fig. 5. (a) Side-view SEM of a silica microtoroid. (b) Top view photomicrograph of the same microtoroid coupled to a fiber taper. (c) Schematic diagram of the typical experimental arrangement for testing OMO [23].

with $Q_{\text {mech }}=2100$ and $m_{\text {eff }}=2.3 \times 10^{-11} \mathrm{~kg}$. As an aside, for $\Omega \ll 2 \delta$ [12], [13], the maximum gain (minimum $P_{\text {th }}$ ) occurs around $\Delta_{0} \sim \delta$. Fig. 5(c) shows the schematic diagram of a typical experimental arrangement for characterizing optomechanical oscillations of a silica microtoroid. A tunable laser with a linewidth smaller than the linewidth of the optical resonance is coupled to the microtoroid via a silica fiber taper. Optical detuning $\left(\Delta_{0}\right)$ is controlled by tuning the laser wavelength, and optical coupling (that determines $Q_{\mathrm{L}}$ ) is controlled by tuning the coupling gap (using a nanopositioner).

The output optical power is detected by a photodetector with a bandwidth larger than $f_{\mathrm{OMO}}$. The photocurrent is fed to an oscilloscope that monitors the temporal behavior of the OMO, an RF spectrum analyzer that monitors the output spectrum, and a phase noise analyzer that measures the oscillation phase noise.

\section{B. Optomechanical Oscillation Frequency}

The intrinsic resonant frequency of mechanical modes of silica microtoroids $\left(f_{\text {mech }}=\Omega_{0} / 2 \pi\right)$ are determined by the geometrical parameters, mode order, and ambient temperature. Calculated values of $f_{\text {mech }}$ using the finite-element method (FEM) are in very good agreement with measured values [12]. As expected, larger values of $f_{\text {mech }}$ can be obtained by reducing $D$, $L$, and $d$; however, the minimum diameter required for maintaining large optical- $Q$ (without introducing radiation loss) sets an upper limit on $f_{\text {mech }}$. To increase the oscillation frequency beyond this limit, high-order mechanical modes can be employed. Using this approach, gigahertz rate oscillation has been obtained in silica microspheres on a silicon pillar [31] (using a fabrication process identical to that used for microtoroids). The optomechanical oscillation frequency $\left(f_{\mathrm{OMO}}\right)$ is offseted from $f_{\text {mech }}$ by optical spring effect [28], [29] and the optical absorption [23]. The optical spring effect offsets $f_{\text {OMO }}$ by $\Delta \Omega_{\mathrm{OM}}$, while absorption of circulating optical power affects $f_{\text {OMO }}$ through internal heat generation that modifies $f_{\text {mech }}$ (intrinsic frequency in the absence of optical power). Since both circulating optical power and $\Delta \Omega_{\mathrm{OM}}$ depend on $\Delta_{0}, f_{\mathrm{OMO}}$ will 


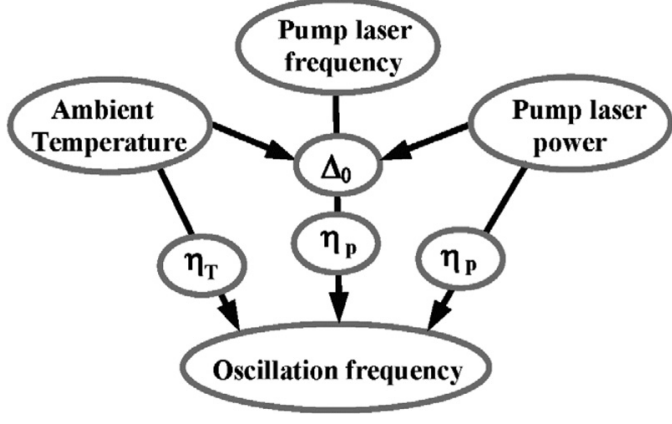

Fig. 6. Schematic diagram showing the hierarchy of the parameters that control the optomechanical oscillation frequency.

be susceptible to variation of optical detuning. These effects can be summarized as follows [23]:

$$
\begin{aligned}
\Omega & =\Omega_{0}\left(1+\eta_{P}\left(\Delta_{0}\right) P\right) \\
\eta_{P}\left(\Delta_{0}\right) & =K_{\mathrm{os}}\left(\Delta_{0}\right)+K_{\mathrm{Th}}\left(\Delta_{0}\right)
\end{aligned}
$$

where $K_{\mathrm{OS}}\left(=\Delta \Omega_{\mathrm{OM}} /\left(P \Omega_{0}\right)\right.$ [see (4)] and $K_{\mathrm{Th}}$ are the proportionality factors for the optical spring and the optical absorption effect, respectively. $K_{\mathrm{Th}}$ is determined by the geometry and dynamics of the heat flow within the microtoroid. Clearly, the oscillation frequency of the OMO also depends on the ambient temperature. Indeed, in the absence of applied optical power, the principal external parameter determining the oscillation frequency of each mechanical eigen mode $\left(\Omega_{0}\right)$ is the ambient temperature. In the linear regime $\left(\Delta T / T_{0} \ll 1\right)$, this thermal effect may be summarized as

$$
\Omega_{0}^{\prime}=\Omega_{0}\left(1+\eta_{T}\left(T-T_{0}\right)\right)
$$

where $\eta_{T}$ is the thermal coefficient of the corresponding optomechanical mode. In conclusion, the frequency variations in an OMO are caused by changes in the ambient temperature, pump laser power $(P)$, and pump laser frequency $\left(\omega_{0}\right)$. The ambient temperature affects the oscillation frequency by changing the natural mechanical resonant frequency $\left(f_{\text {mech }}=\right.$ $\left.\Omega_{0} / 2 \pi\right)$ as well as the optical frequency detuning $\left(\Delta_{0}\right)$. The pump laser power $(P)$ and frequency affect the oscillation frequency through the optical spring effect and optical absorption. The fast variations of laser power and frequency (relative intensity noise (RIN) and frequency jitter) manifest themselves in the phase noise of the optomechanical oscillations, while their slow variations result in optomechanical oscillation frequency drift. Fig. 6 summarizes the relation among these parameters.

Usually, the slow variations of laser power and frequency are stabilized by feedback circuitry inside the laser. Furthermore, it has been shown that the slow variations of the optical frequency detuning $\left(\Delta_{0}\right)$ can be stabilized using an external feedback circuit that controls the laser wavelength to compensate for the optical resonant frequency drift [32]. Using (6)-(8), the fractional frequency change due variations in optical pump power, optical frequency detuning, and temperature variations can be expressed as

$$
\frac{\delta \Omega}{\Omega_{0}}=\eta_{p}(\Delta) \delta P+P \frac{d \eta_{p}}{d(\Delta)} \delta(\Delta)+\eta_{T} \delta T
$$

where $\delta$ denotes small variations of the corresponding parameter. As mentioned previously, $\eta_{p}\left(\Delta_{0}\right)$ and $\eta_{T}$ are determined by the geometry of the resonator, and hence, can be tailored through careful design. For the fourth mechanical mode of the microtoroid in Fig. 5(a), $\Omega_{0}=54200 \mathrm{kHz}, \eta_{T} \sim 1.3 \times$ $10^{-4} \mathrm{~K}^{-1}, \eta_{p}(\delta) \sim 1.5 \times 10^{-6} \mu \mathrm{W}^{-1}$, and $d \eta_{p} / d \Delta_{0}=4.8 \times$ $10^{-3} \mu \mathrm{W}^{-1} \mathrm{pm}^{-1}[23]$.

\section{Phase Noise: Classical (Brownian noise) and Quantum Mechanical (Back-Action Noise)}

Phase noise is one of the most important characteristics of any self-sustained oscillator. The phase noise spectral density of a microtoroid OMO exhibits a dependence of $1 / f^{3}$ at offset frequencies between $100 \mathrm{~Hz}$ and $10 \mathrm{KHz}$, and a dependence of $1 / f^{2}$ at offsets larger than $10 \mathrm{KHz}$. The $1 / f^{3}$ regime is a signature of $1 / f$ or "flicker noise" in the corresponding frequency fluctuation spectrum, while the appearance of $1 / f^{2}$ regime is mainly due to the presence of the white noise in this spectrum. The $1 / f$ components are currently not well understood. However, the white noise is known to be related to the random Langevin force $\left[F_{\mathrm{L}}(\mathrm{t})\right.$ in (1)] and determines the short-term oscillation linewidth $\left(\Delta \Omega_{\mathrm{OMO}}\right)$. At room temperature, and for typical values of $Q_{\mathrm{o}}$ and $Q_{\mathrm{mech}}$, the linewidth of the radiationpressure-driven OMO may be explained using the general theory of line narrowing in self-sustained oscillators, which has been developed in the context of optical oscillators (SchawlowTownes line narrowing in lasers) as well as electronic oscillators [33], [34]. All oscillators that are fundamentally limited by a white noise source (spontaneous emission in lasers and Johnson noise in electronic oscillators) are essentially governed by similar differential equations with the only differences in the physical interpretation of the coefficients and noise terms. In a thermally limited oscillator, this theory states that the oscillation linewidth $\left(\Delta \Omega_{\mathrm{OMO}}\right)$ is inversely proportional to the oscillator output power $\left(P_{d}\right)$ such that [35]

$$
\Delta \Omega_{\mathrm{OMO}}=\frac{\boldsymbol{k}_{B} \boldsymbol{T}}{2 \boldsymbol{P}_{\boldsymbol{d}}}\left(\Delta \Omega_{0}\right)^{2}
$$

where $\Delta \Omega_{0}=\gamma_{0}=\Omega_{0} / Q_{\text {mech }}$ is the intrinsic linewidth of the mechanical mode. Through a detailed study of the shortterm stability of the OMO, it has been demonstrated that in the above-threshold regime, the measured mechanical oscillation linewidth is in good agreement with (10) [35]. In particular, at room temperature, the thermomechanical noise, also referred to as Brownian noise, is the dominant noise mechanism in the microtoroid OMO. For an OMO, (10) can be rewritten as

$$
\Delta \Omega_{\mathrm{OMO}}=\left(\frac{4 k_{B} \boldsymbol{T} \boldsymbol{Q}_{\mathrm{L}}^{2}}{\boldsymbol{m}_{\mathrm{eff}} \Omega_{0}^{2} \boldsymbol{R}_{0}^{2}}\right) \frac{\Gamma_{\Omega}^{2} \Delta \Omega_{0}}{\boldsymbol{M}^{2}}
$$

where $M$ is the measured optical modulation depth of the optical output power (induced by resonator motion) and $\Gamma_{\Omega}$ is the optical modulation transfer function [23]. Validation of this equation is a necessary and sufficient condition for identifying thermal noise as the fundamental limit for the oscillation linewidth. Fig. 7 shows the measured oscillation linewidth versus optical modulation depth $(M)$ for the OMO in Fig. 5(a). 


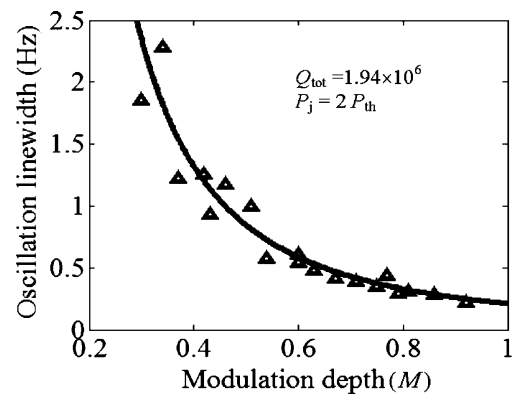

Fig. 7. Measured oscillation linewidth plotted versus modulation depth $(M)$. The solid line is the calculated linewidth using (11) [23].

The solid line is the theoretical prediction obtained from (11) $\left(m_{\text {eff }} \sim 2.3 \times 10^{-11} \mathrm{~kg}, \Delta \omega \approx \delta, \Gamma_{\Omega} \sim 0.55, Q_{\text {mech }}=2100\right.$, and ambient temperature). The good agreement between theoretical prediction and the experimental result proves that the fundamental limit for the short-term linewidth of a microtoroid OMO is actually the thermal noise. Long-term phase noise would be influenced also by the $1 / f$ (jitter) components.

While at room temperature, the linewidth (phase noise) scales inversely with oscillator energy and linearly with temperature (in accordance with the classical theory of phase noise in a regenerative system), and quantum back action sets a fundamental limit to its linewidth at low temperature and high mechanical$Q$ [36]. In this context, the quantum back action refers to the noise in the radiation pressure generated by the granularity of photons (photon shot noise). In other words, the spectral purity, as measured by the phase noise, has a back-action contribution (in addition to the classical Brownian noise contribution). It has been shown that this quantum back action component of the linewidth can be written as [36]

$$
\Delta \Omega=\frac{\gamma_{0} n_{T}}{\bar{n}_{c}}+\frac{A_{+}}{2 \bar{n}_{c}}
$$

where $n_{T}$ is the thermal occupation at temperature $T$ and at the mechanical oscillation frequency, $n_{c}$ is the number of phonons in the coherently oscillating mechanical mode, and $\mathrm{A}_{+}$is the Stokes rate [36]. The first term in (12) is the classical contribution to the oscillator linewidth discussed before, while the second term is the contribution of quantum back action to the oscillation phase noise.

\section{Mechanical-Q: Effect of Pressure and Geometry}

The magnitude of $P_{\mathrm{th}}$ and the high-frequency phase noise of an OMO are inversely proportional to the quality factor of the mechanical resonator [see (6) and (10)]. Therefore, improving the mechanical- $Q\left(Q_{\text {mech }}\right)$ significantly improves the performance of the OMO. The quality factor of the mechanical modes of a silica microtoroid is limited by three major mechanisms: clamping loss (mechanical energy loss of the silica membrane to the silicon pillar), internal friction in silica, and external friction due to presence of air molecules. The contribution of these mechanisms depends on the geometrical parameters of the microtoroid ( $D, L, t$, and $d$ ), as well as the order of the mechanical mode that defines its motion. A comprehensive characteriza-

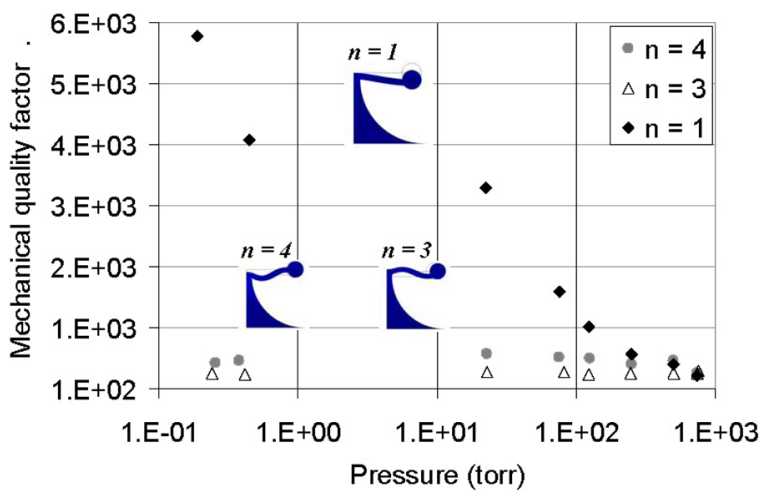

Fig. 8. Changes of mechanical- $Q$ for a silica microtoroid. The insets are the corresponding mechanical modes [unpublished results from the authors].

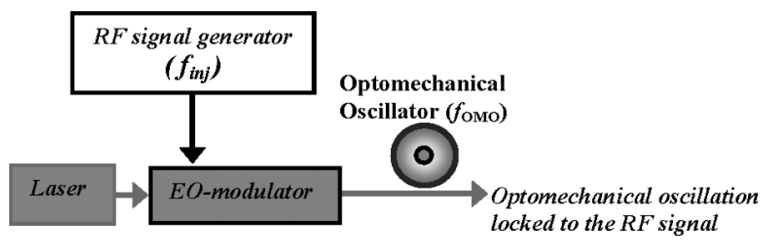

Fig. 9. Injection locking of OMO to an external electronic oscillator.

tion of $Q_{\text {mech }}$ in microtoroids is a very difficult task due to involvement of various coupled parameters, as well as the mode dependence. Studies have been limited to certain modes and rely mainly on experimental observations rather than theory. A detailed study of mechanical- $Q$ in silica microtoroids, as well as a modified silica microtoroid structure, with significantly larger $Q_{\text {mech }}$ compared to conventional microtoroids is presented in [37]. The contribution of clamping losses can be studied by varying the diameter of the silicon pillar holding the silica resonator [37]. The effect of ambient pressure on $Q_{\text {mech }}$ of a mechanical mode strongly depends on the mechanical-mode deformation [38]. Fig. 8 provides the measured variation of $Q_{\text {mech }}$ for three mechanical modes $\left(n=1\right.$ with $f_{\text {mech }}=3.6 \mathrm{MHz}, n=$ 3 with $f_{\text {mech }}=22.4 \mathrm{MHz}$, and $n=4$ with $f_{\text {mech }}=63.2 \mathrm{MHz}$ ) as ambient pressure is changed from 760 to 0.1 torr. Among these, only $n=1$ (the flexural mode) experiences significant resistance from air molecules, and, as expected, its quality factor improves as the ambient pressure is lowered.

\section{E. Injection-Locked OMO}

When a periodic signal with a frequency close to the oscillation frequency and large enough amplitude is injected into a self-sustained oscillator, the phase and frequency of the oscillator can be locked to that of the injected signal. This phenomenon known as "injection locking" can be used to lock the frequency and phase of the OMO to that of an external oscillator [39]. Basically, partial amplitude modulation of the optical input power can lock both frequency and phase of the optomechanical oscillation to that of the external oscillator used to modulate the input optical power. This can be another OMO, an electronic oscillator, or an optoelectronic oscillator [40]. Fig. 9 shows a 


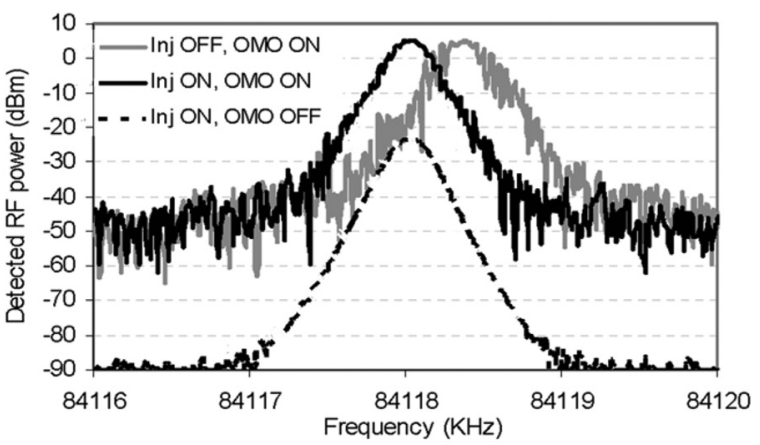

(a)

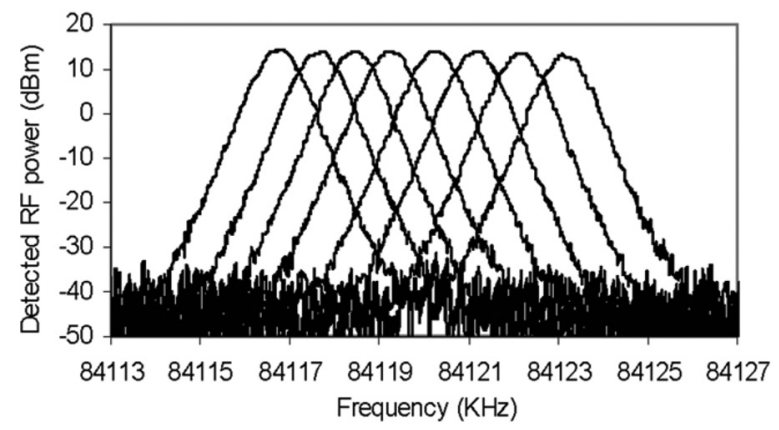

(b)

Fig. 10. (a) RF spectrum of the optical output power in the presence (black) and absence (gray) of the injection signal. The dashed trace is the spectrum of the optical input power. (b) RF spectrum of the optomechanical oscillation tuned by injection signal [39].

possible configuration for injection locking of OMO. Here, the optical input power $(P)$ is partially modulated by a single-tone RF signal from an RF source applied on a Mach-Zehnder (MZ) modulator. The modulation index is thereby used to control the strength of the weaker injection-locking signal (modulated fraction of $P$ ) in comparison to the pump (continuous wave $(\mathrm{CW})$ fraction of $P$ ).

The laser power and amplitude modulation depth should be chosen such that the $\mathrm{CW}$ optical input power is larger than $P_{\text {th }}$, but small enough to keep the oscillation in the linear regime. Fig. 10(a) shows the RF spectrum of the optical output power of an OMO in the absence (gray trace) and presence (black trace) of modulation (injection) [39]. The dashed trace is the spectrum of the optical input power (measured when the OMO is decoupled from the waveguide). Here, $f_{\mathrm{OMO}}=84.1 \mathrm{MHz}$ and the frequency of the injected signal $\left(f_{\text {inj }}\right)$ is $500 \mathrm{~Hz}$ smaller than the optomechanical oscillation frequency. It is apparent that the presence of the modulation pulls $f_{\text {OMO }}$ toward $f_{\text {inj }}$ and locks the two signals. As shown in Fig. 10(b), injection locking can be used to tune the optomechanical oscillation frequency simply by tuning $f_{\text {inj }}$. Experimental results confirm that injection locking in an OMO exhibits the characteristics of injection locking in electronic oscillators [39]. At a given ratio between modulation amplitude of the input signal and amplitude of the optomechanical oscillation imposed on the optical output $\left(A_{\mathrm{inj}} / A_{\mathrm{OMO}}\right)$, locking occurs within a limited bandwidth around $f_{\mathrm{OMO}}$ (known as lock range).

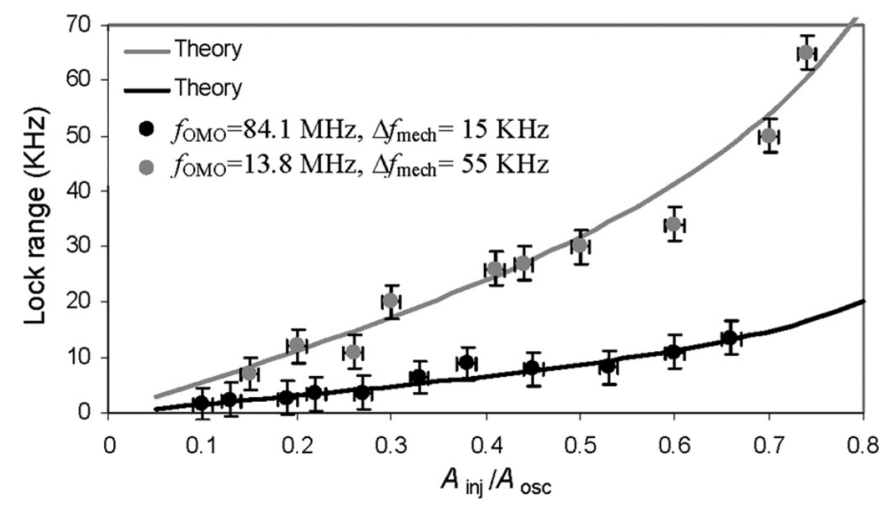

Fig. 11. Measured lock range plotted against the ratio between the modulation amplitude and optomechanical oscillation amplitude $\left(A_{\text {inj }} / A_{\mathrm{OMO}}\right)$ for two different OMOs [39]. The solid lines are the theoretical estimations based on (13). $\Delta f_{\text {mech }}$ is the intrinsic linewidth of the passive mechanical resonator.

Based on the theoretical prediction using the general theory of injection locking for self-sustained oscillators, the lock range can be written as

$$
\Delta f_{\text {lock }}=\Delta f_{\text {mech }} \frac{A_{\text {inj }}}{A_{\mathrm{OMO}}}\left(1-\left(\frac{A_{\text {inj }}}{A_{\mathrm{OMO}}}\right)^{2}\right)^{-1 / 2}
$$

where $\Delta f_{\text {mech }}=\Delta \Omega_{0} / 2 \pi=f_{\text {mech }} / Q_{\text {mech }}$ is the intrinsic linewidth of the passive mechanical resonator. Beyond the lock range, the phase difference between optomechanical oscillation and the injected signal becomes random and the two signals are unlocked. Fig. 11 shows the measured lock range as a function of $A_{\text {inj }} / A_{\text {OMO }}$ for two different OMOs: one with $f_{\text {OMO }}=$ $13.8 \mathrm{MHz}$ and $\Delta f_{\text {mech }}=55 \mathrm{kHz}$, and the other with $f_{\mathrm{OMO}}=$ $84.1 \mathrm{MHz}$ and $\Delta f_{\text {mech }}=15 \mathrm{kHz}$. The solid lines are the theoretical predictions based on (13).

It has been shown that the presence of a low, phase-noise locking signal also improves the magnitude of the OMO phase noise proportional to $A_{\mathrm{inj}} / A_{\mathrm{OMO}}$. Moreover, it has been verified that, as is also true with electronic oscillators, the OMO can be injection-locked to a signal with a frequency close to harmonic or subharmonic frequencies of the fundamental oscillation frequency $\left(f_{\text {OMO }}\right)$ [39]. The optomechanical injection locking is a useful feature that can expand the applications of OMO. Injection locking of an OMO to an electronic RF oscillator enables combination of OMO with electronic devices, and may have interesting applications in certain RF photonic and microoptoelectromechanical systems (MOEMs). In this context, injection locking may be used to lock two OMOs, and improve the frequency stability and noise performance of the "slave" oscillator similar to what is done in the optical domain with lasers (improving the performance of a noisy high-power laser with a narrow linewidth low-power laser). Lastly, injection locking is a useful feature in metrological applications where it can be used to synchronize multiple OMOs as well as controlling optomechanical oscillations with atomic accuracy (by locking to atomic clocks). 


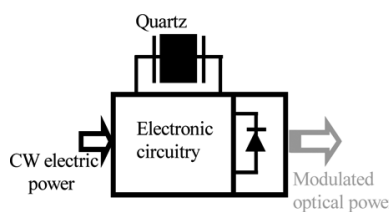

(a)

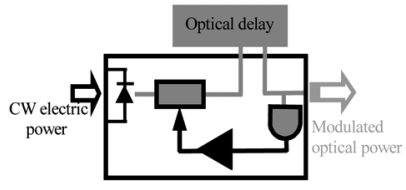

(b)

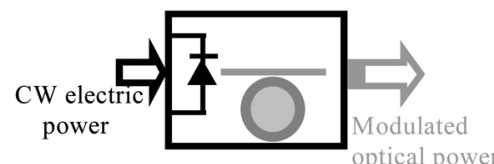

(c)

Fig. 12. Alternative configurations for a photonic clock. (a) Laser diode driven by a quartz oscillator (electomechanical). (b) Optoelectronic oscillator [40]. (c) OMO.

\section{Potential ApPlications in RF Photonics}

\section{A. OMO as a Photonic Clock}

The radiation-pressure-driven OMO is essentially an RF (10-200 MHz) photonic clock that has the potential to be integrated with photonic circuits and devices. A photonic frequency reference or photonic clock can be defined as a device that can generate a stable harmonically amplitude-modulated optical field. Although one may use a standard electronic clock to modulate a laser source and generate the optical clock signal, here we use the term photonic clock exclusively when the optical field is directly involved in the feedback mechanism that sustains the oscillatory motion. A well-known example of such device is an optoelectronic oscillator [40]. The optoelectronic oscillator functions based on electrooptic interaction where the feedback loop consist of an intensity modulator, optical fiber delay line, a photodetector, an amplifier, and a filter. In an OMO, the feedback is intrinsically provided through the optomechanical coupling and the optical resonance. The intrinsic feedback mechanism of an OMO results in an exceptional simplicity, size reduction, and power efficiency that is hard to achieve not only with optoelectronic oscillators but also with conventional electronic devices. Fig. 12 shows three possible techniques that can be used for generation of a harmonically modulated optical output power. Clearly, among these configurations, the OMO provides the simplest solution with the smallest footprint.

\section{B. Nonlinearity of Optomechanical Oscillation and RF Mixing}

The optical transfer function of the OMO is a Lorentzian, and therefore, its behavior is linear only in the small-signal regime and for a detuning $\left(\Delta_{0}\right)$ close to $50 \%$ of the optical resonant linewidth $(\delta)$. For large signals and $\Delta_{0} \neq \delta$, optomechanical modulation of the optical resonance results in nonlinear amplitude modulation of the transmitted optical power [23]. The nonlinearity of the optomechanical transfer function manifests itself through the appearance of harmonics of the mechanical eigen frequencies within the spectrum of the modulated optical power. The strength of the nonlinear behavior for an OMO is controlled by $P$ and $\Delta_{0}$ [23], [24]. For applications where a single-tone oscillation is required, these parameters may be adjusted to obtain linear optomechanical oscillation. Nonlinear

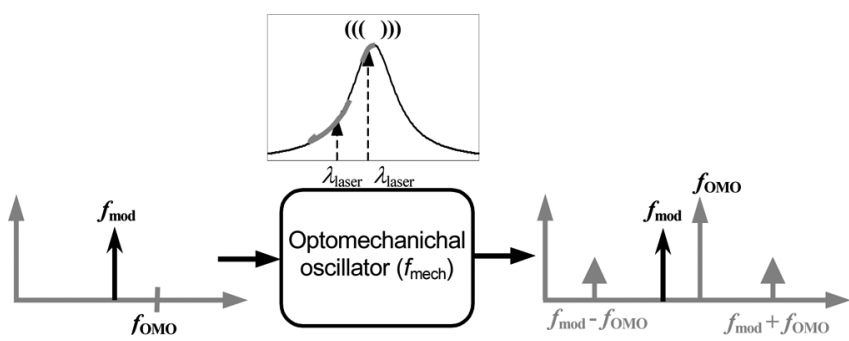

Fig. 13. Concept of frequency mixing in an OMO.

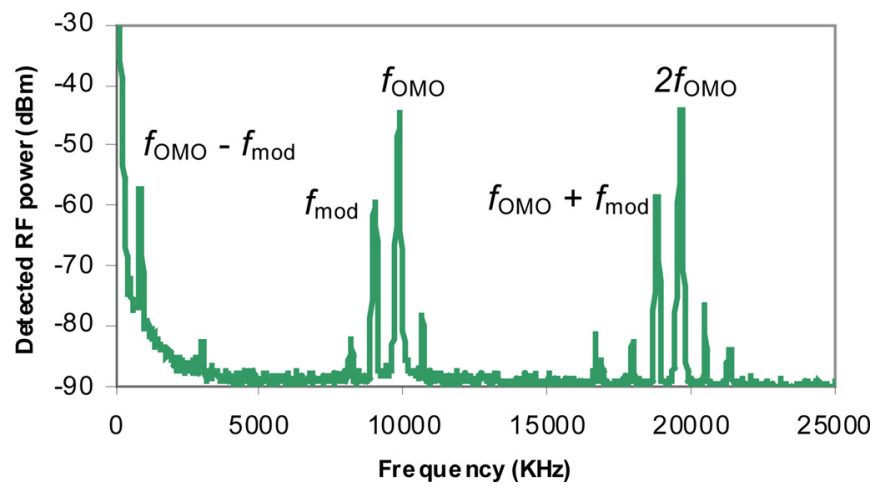

Fig. 14. Measured output spectrum of an OMO with $f_{\mathrm{OMO}}=10 \mathrm{MHz}$ and with an optical input power modulated at $f_{\mathrm{mod}}=1 \mathrm{MHz}$ [source: unpublished results from authors].

behavior on other hand can be beneficial for applications where $\mathrm{RF}$ mixing in photonic domain is desired (RF photonic signal processing). Fig. 13 shows the concept of optomechanical RF mixing. If one modulates the amplitude of the optical input pump power $(P)$ at a frequency $f_{\text {mod }}$ and tunes the laser wavelength to the nonlinear region of the optical transfer function (shown as gray sections), the up- and down-converted signals ( $f_{\mathrm{OMO}} \pm$ $f_{\text {mod }}$ ) will appear in the spectrum of the optical output power.

These frequency components are generated through the optomechanical oscillation $\left(f_{\mathrm{OMO}}\right)$ of the nonlinear transfer function in the presence of externally modulated $\left(f_{\mathrm{mod}}\right)$ optical pump power. Fig. 14 shows the measured output spectrum of an OMO with $f_{\mathrm{OMO}}=10 \mathrm{MHz}$ and optical input power modulated at $f_{\text {mod }}=1 \mathrm{MHz}$. It is important to note that here we assume that during the modulation period $\left(1 / f_{\bmod }\right), P$ is large enough to compensate for reduction of optomechanical gain caused by tuning the laser to the nonlinear region of the transfer function.

\section{Optomechanical Homodyne RF Receiver}

Combining the RF mixing capability of OMO with its highquality oscillatory properties makes it a useful device for photonic RF receiver applications. Basically, the OMO can simultaneously function as the nonlinear element and the local oscillator (LO) to down convert the baseband signal from a standard suppressed-carrier RF signal. In an electronic homodyne receiver [see Fig. 15(a)], the baseband signal is down converted from the RF carrier by mixing the received signal with an LO that has the same frequency as the RF carrier $\left(f_{\mathrm{RF}}\right)$. In a conventional system, the mixing occurs through the nonlinearities of electronic elements (such as diodes and transistors). In addition, 


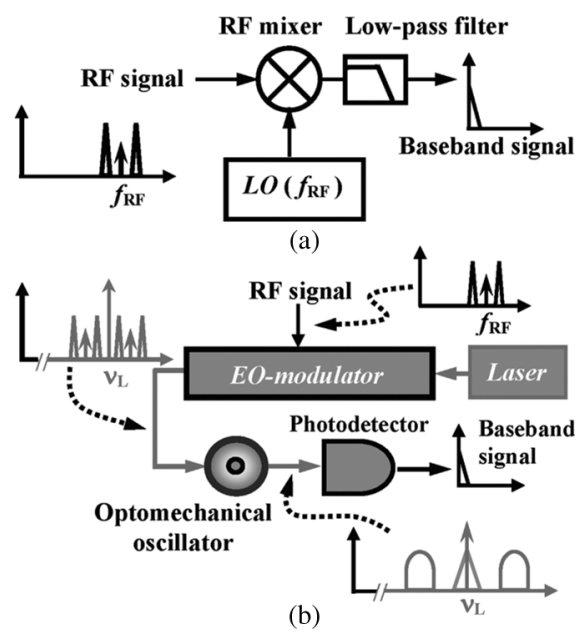

Fig. 15. (a) Schematic diagram of a standard electronic homodyne RF down converter. (b) Schematic diagram of the photonic RF down converter based on microtoroidal OMO and the RF/optical spectrum of the signal while it flows through the system (indicated by dotted arrows).

the LO uses electronic components as well as an electromechanical reference resonator such as quartz crystal. In the proposed architecture [see Fig. 15(b)], the received RF signal modulates the amplitude of an optical carrier $\left(\nu_{\mathrm{L}} \approx 194 \mathrm{THz}\right)$ in an electrooptic modulator. The modulated optical wave is then fed to a microtoroidal silica resonator, and finally, the transmitted optical power is detected in a photodetector with a bandwidth matched to the baseband frequency. When the optical input power to the microtoroid is larger than $P_{\text {th }}$ and $f_{\mathrm{OMO}}$ is equal to the RF carrier frequency $\left(f_{\mathrm{RF}}\right)$, the optical mixing inside the OMO generates a baseband component on the optical carrier, and therefore, upon detection, the photocurrent will be baseband-modulated (the high-frequency RF components are filtered out through the slow response of the photodetector).

In other words, the optomechanical oscillation combined with the nonlinear optical transfer function of the microtoroid (Lorentzian) reproduce the combined function of the $\mathrm{LO}$ and the mixer in an electronic down converter [see Fig. 15(a)]. Note that the bandwidth of the loaded optical resonance imposes an upper limit on the maximum frequency of the baseband signal. Fig. 16(a) shows the diagram of the experimental setup used for the proof-of-concept demonstration [24].

The RF signal is an RF carrier $(25.11 \mathrm{MHz})$ modulated by a single-tone $\left(f_{S}=1 \mathrm{MHz}\right)$ baseband signal (with suppressed carrier modulation format). This signal modulates the output of a tunable laser $(\lambda \sim 1550 \mathrm{~nm})$ in a Mach-Zehnder modulator. Here, $P_{\mathrm{th}}=120 \mu \mathrm{W}, P=270 \mu \mathrm{W}$, and $f_{\mathrm{OMO}}=$ $25.11 \mathrm{MHz}$. If the laser wavelength is tuned off-resonance, the modulated optical wave is transmitted through the fiber-taper without being coupled to the microtoroid, and the RF spectrum of the detected optical power is identical to the spectrum of a suppressed carrier-modulated RF wave or the signal that is driving the MZ modulator [see gray trace in Fig. 16(b) and (c)]. When the laser is tuned inside the optical resonance, the circulating optical power initiates the self-sustained optomechanical oscillation that boosts the modulated RF power at $25.11 \mathrm{MHz}$ [see black trace in Fig. 16(b)]. Meanwhile, since the laser is

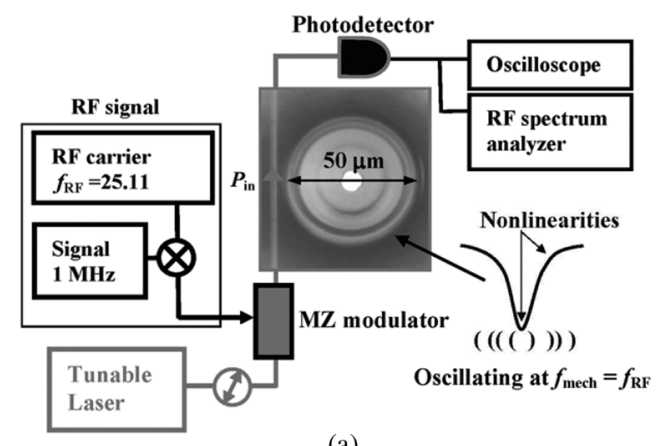

(a)

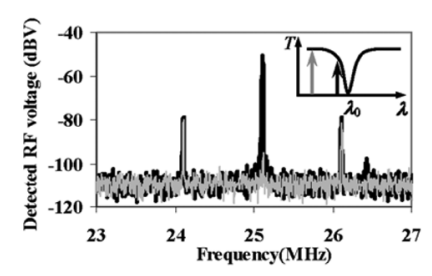

(b)

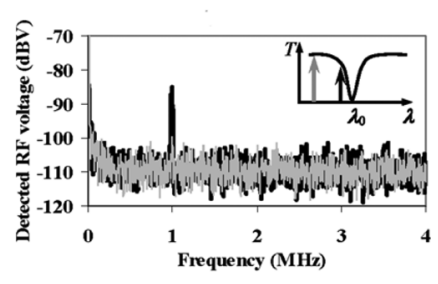

(c)
Fig. 16. (a) Schematic diagram of the experimental setup. The chosen optical mode of the microtoroid has an intrinsic and loaded optical quality factor of $1 \times 10^{7}$ and $0.5 \times 10^{7}$, respectively. The mechanical mode has a frequency of $25.11 \mathrm{MHz}$ and quality factor of about $Q_{\text {mech }} \sim 4000$. Off-resonance (gray) and on-resonance (black) RF spectrum of the transmitted optical power (b) near $f_{\mathrm{RF}}$ and (c) near the baseband frequency. The insets show the relative detuning of the laser wavelength and the optical resonance for each case ( $T$ is the transmitted optical power) [24].

tuned to the nonlinear section of the optical transfer function, the oscillation mixes with the RF sidebands $\left(f_{\mathrm{OMO}} \pm f_{S}\right)$ and down converts the single-tone baseband $\left(f_{S}\right)$ [see black trace in Fig. 16(c)]. Note that previously self-homodyne RF photonic receivers were demonstrated based on nonlinear modulation (in an electrooptic microdisk) and optical filtering [41], [42]. However, in these architectures, the LO was eliminated using a transmitted carrier modulation format, and as a result, they suffered from low power efficiency as well as incompatibility with standard RF transceivers for long-distance communication. In contrast, the dual functionality of the OMO (oscillator/mixer) makes it compatible with suppressed carrier modulation format. At this stage, the optomechanical down converter may not be a feasible replacement for its electronic counterparts; however, the fundamental idea lays the foundation for further research in this relatively young field.

\section{Frequency Mixing in Subcarrier Optical Links}

In RF subcarrier optical links, the optomechanical RF mixing can be used to selectively down convert the baseband information from the RF carrier based on the wavelength of the optical carrier. Fig. 17 shows one possible configuration where three optical carriers each carrying an RF signal with a different RF carrier frequency are multiplexed through a fiber optical link. At the destination, the three microtoroid OMOs with frequencies matched to the RF carrier frequencies are coupled to the fiber.

If the optical resonance of each OMO is also matched to the corresponding optical carrier when the optical output is demultiplexed, each optical wavelength will be modulated with the 


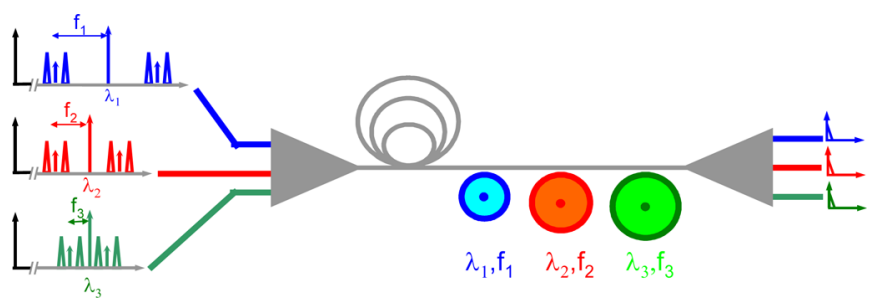

Fig. 17. Schematic diagram of a RF subcarrier link employing OMOs for wavelength selective baseband down-conversion in photonic domain.

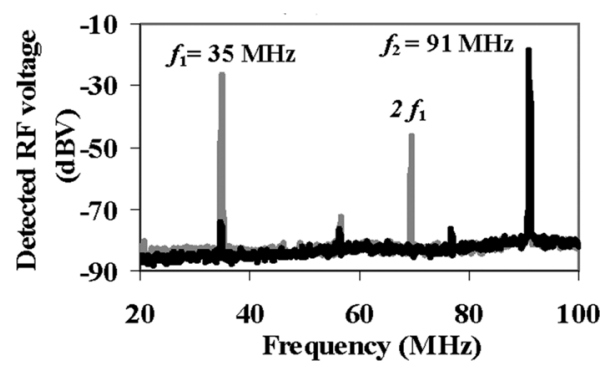

Fig. 18. RF spectrums of the detected optical output power at two different laser wavelength detunings (the black and gray traces) each exciting a different mechanical mode [24].

baseband carried by its RF carrier. Therefore, the data from each channel are down-converted without using any electronic element. Usually, at a given optical input power and optical coupling strength, the microtoroid oscillates at one of its eigen mechanical modes. However, by tailoring the geometry of the microtoroid and adjusting the optical coupling factor, it is possible to tune the $P_{\text {th }}$ for two (or even 3) different eigen mechanical modes such that each one falls below $P$ at a distinct optical detuning $\left(\Delta_{0}\right)$. As a result, the OM oscillation frequency can be switched only by changing $\Delta_{0}$ (tuning the laser wavelength)). Fig. 18 shows the RF spectrums of the detected optical output power of an $\mathrm{OMO}$ at two different laser wavelength detunings: $\Delta_{01} \sim 0.3 \delta$ (gray trace) and $\Delta_{02} \sim 0.7 \delta$ (black trace) [24]. The fundamental $\left(f_{1}=35 \mathrm{MHz}\right)$ and third $\left(f_{2}=91 \mathrm{MHz}\right)$ mechanical eigen frequencies of the microtoroid have been selectively excited only by adjusting $\Delta_{0}$ to $\Delta_{01}$ and $\Delta_{02}$, respectively. This degree of freedom could be useful in the context of the downconverting RF optical links since it enables data down conversion from two distinct RF carrier frequencies only by tuning the laser wavelength within the optical resonance. The concept of wavelength-dependent optomechanical oscillation frequency can be used to replicate the function of two OMOs in the optical link shown in Fig. 17 with a single OMO.

\section{E. Future Prospects for Integration}

Monolithic integration of microtoroid OMOs with conventional silicon photonics devices is a challenging task due to their special fabrication process and geometry. However, rapid progress in microresonator research suggests that devices featuring performance comparable to microtororoids, fabricated using techniques compatible with integrated silicon photonics, will one day be possible. For example, optical- $Q$ factors as high as 50 million have already been obtained without silica reflow [45].

Ultimately, however, novel OMOs must be developed that are more compatible with existing silicon photonic platforms. Recently, two novel optomechanical resonators have been demonstrated: suspended waveguide and suspended photonic crystal optomechanical resonators [43], [44]. The suspended waveguide is made of silicon and suspended photonic crystal is made of silicon nitride using standard CMOS-compatible processes. With further development and optimization, these devices may become efficient enough to replicate the optomechanical functions demonstrated using silica microtoroids. Meanwhile the silica microtoroid OMO, that fulfills all requirements of efficient optomechanical oscillation, will serve as an ideal platform for studying the physics of optomechanical oscillation and exploring its potential engineering applications.

\section{CONCLUSION}

In summary, we have described the physics, fundamental characteristic, and potential applications of the optomechanical oscillations in the context of silica microtoroids. With recent advancements in microfabrication techniques, the performance of a microtoroidal OMO may be improved to a level that it competes with its electronic counterparts. Novel approaches are also underway that may overcome the frequency limits mentioned here. Although a fully integrated OMO on a silicon chip has not been realized yet, the materials and techniques used in fabricating OMOs are highly compatible with conventional materials and techniques used in MEMS and IC industry. The effort toward design and fabrication of fully integrated optomechanical systems has been already started [43], [44]. These systems are potential candidates for integration with existing photonic and electronic platforms.

\section{REFERENCES}

[1] V. B. Braginski and A. B. Manukin, "Ponderomotive effects of electromagnetic radiation," Soviet Phys. JETP, vol. 25, no. 4, pp. 653-655, 1967.

[2] V. B. Braginski, A. B. Manukin, and M. Y. Tikhonov, "Investigation of dissipative ponderomotive effects of electromagnetic radiation," Soviet Phys. JETP, vol. 31, no. 5, pp. 829-830, 1970.

[3] V. B. Braginsky, Measurement of Weak Forces in Physics Experiments. Chicago, IL: Univ. of Chicago Press, 1977.

[4] C. M. Caves, K. S. Thorne, R. W. P. Drever, V. D. Sandberg, and M. Zimmermann, "On the measurement of a weak classical force coupled to a quantum-mechanical oscillator," Rev. Mod. Phys., vol. 52, no. 2, pp. 341-392, 1980.

[5] C. M. Caves, "Quantum-mechanical noise in an interferometer," Phys. Rev. D, vol. 23, no. 8, pp. 1693-1708, 1981.

[6] A. Dorsel, J. D. McCullen, P. Meystre, E. Vignes, and H. Walther, "Optical bistability and mirror confinement induced by radiation pressure," Phys. Rev. Lett., vol. 51, no. 17, pp. 1550-1553, 1983.

[7] V. B. Braginsky and S. P. Vyatchanin, "Low quantum noise tranquilizer for Fabry-Perot interferometer," Phys. Lett. A, vol. 293, pp. 228-234, 2002.

[8] M. I. Dykman, "Heating and cooling of local and quasi local vibrations by a nonresonant field," Sov. Phys. Solid State, vol. 20, pp. 1306-1311, 1978.

[9] V. B. Braginsky, S. E. Strigin, and S. P. Vyatchanin, "Parametric oscillatory instability in Fabry-Perot interferometer," Phys. Lett. A, vol. 287, pp. 331338, 2001. 
[10] H. Rokhsari, T. J. Kippenberg, T. Carmon, and K. J. Vahala, "Radiationpressure-driven micro-mechanical oscillator," Opt. Exp., vol. 13, no. 14, pp. 5293-5301, 2005.

[11] T. Carmon, H. Rokhsari, L. Yang, T. J. Kippenberg, and K. J. Vahala, "Temporal behavior of radiation-pressure-induced vibrations of an optical microcavity phonon mode," Phys. Rev. Lett., vol. 94, p. 223902, 2005.

[12] T. J. Kippenberg, H. Rokhsari, T. Carmon, A. Scherer, and K. J. Vahala, "Analysis of radiation-pressure induced mechanical oscillation of an optical microcavity," Phys. Rev. Lett., vol. 95, p. 033901, 2005.

[13] T. J. Kippenberg and K. J. Vahala, "Cavity opto-mechanics," Opt. Exp, vol. 15, pp. 17172-17205, 2007.

[14] T. J. Kippenberg and K. J. Vahala, "Cavity optomechanics: Back-action at the mesoscale," Science, vol. 321, pp. 1172-1176, 2008.

[15] F. Marquardt, J. G. E. Harris, and S. M. Girvin, "Dynamical multistability induced by radiation pressure in high-finesse micromechanical optical cavities," Phys. Rev. Lett., vol. 96, p. 103901, 2006.

[16] J. D. Thompson, B. M. Zwickl, A. M. Jayich, F. Marquardt, S. M. Girvin, and J. G. E. Harris, "Strong dispersive of a high-finesse cavity to a micromechanical membrane," Nature, vol. 452, pp. 72-75, 2008.

[17] A. Schließer, P. Del'Haye, N. Nooshi, K. J. Vahala, and T. J. Kippenberg, "Radiation pressure cooling of a micromechanical oscillator using dynamical backaction," Phys. Rev. Lett., vol. 97, p. 243905, 2006.

[18] O. Arcizet, P. F. Cohadon, T. Briant, M. Pinard, and A. Heidmann, "Radiation-pressure cooling and optomechanical instability of a micromirror," Nature, vol. 444, no. 7115, pp. 71-74, 2006.

[19] S. Gigan, H. R. Bohm, M. Paternostro, F. Blaser, G. Langer, J. B. Hertzberg, K. C. Schwab, D. Bauerle, M. Aspelmeyer, and A. Zeilinger, "Self-cooling of a micromirror by radiation pressure," Nature, vol. 444, no. 7115 , pp. $67-70,2006$

[20] D. Kleckner and D. Bouwmeester, "Sub-kelvin optical cooling of a micromechanical resonator," Nature, vol. 444, no. 7115, pp. 75-78, 2006.

[21] Schliesser, R. Rivière, G. Anetsberger, O. Arcizet, and T. J. Kippenberg, "Resolved sideband cooling of a micromechanical oscillator," Nature Phys., vol. 4, pp. 415-419, 2008.

[22] C. H. Metzger and K. Karrai, "Cavity cooling of a microlever," Nature, vol. 432, no. 23, pp. 1002-1005, 2004.

[23] M. Hossein-Zadeh, H. Rokhsari, A. Hajimiri, and K. J. Vahala, "Characterization of a radiation-pressure-driven optomechanical oscillator," Phys. Rev. A, vol. 74, no. 2, p. 023813, 2006.

[24] M. Hossein-Zadeh and K. J. Vahala, "Photonic RF down-converter based on optomechanical oscillation," IEEE Photon. Tech. Lett., vol. 20, no. 4, pp. 234-236, Feb. 2008.

[25] C. Metzger, M. Ludwig, C. Neuenhahn, A. Ortlieb, I. Favero, K. Karrai, and F. Marquardt, "Self-induced oscillations in an optomechanical system driven by bolometric backaction," Phys. Rev. Lett., vol. 101, p. 133903, 2008.

[26] K. J. Vahala, "Optical microcavities," Nature, vol. 424, no. 6950, pp. 839846, 2003.

[27] D. K. Armani, T. J. Kippenberg, S. M. Spillane, and K. J. Vahala, "Ultrahigh-Q toroid microcavity on a chip," Nature, vol. 421, no. 6926, pp. 925$928,2003$.

[28] B. S. Sheard, M. B. Gray, C. M. Mow-Lowry, D. E. McClelland, and S. E. Whitcomb, "Observation and characterization of an optical spring," Phys. Rev. A, vol. 69, no. 5, p. 051801, 2004.

[29] M. Hossein-Zadeh and K. J. Vahala, "Observation of optical spring effect in a microtoroidal optomechanical resonator," Opt. Lett., vol. 32, no. 12, pp. 1611-1613, 2007.

[30] M. Cai, O. Painter, and K. J. Vahala, "Observation of critical coupling in a fiber taper to a silica microsphere whispering-gallery mode system," Phys. Rev. Lett., vol. 85, pp. 74-77, 2000.

[31] T. Carmon and K. J. Vahala, "Modal spectroscopy of optoexcited vibrations of a micron-scale on-chip resonator at greater than $1 \mathrm{GHz}$ frequency," Phys. Rev. Lett., vol. 98, p. 123901, 2007.

[32] T. Carmon, T. J. Kippenberg, L. Yang, H. Rokhsari, S. M. Spillane, and K. J. Vahala, "Feedback control of ultra-high-Q microcavities: application to micro-raman lasers and microparametric oscillators," Opt. Exp., vol. 13, pp. $3558-3566,2005$.

[33] A. L. Schawlow and C. H. Townes, "Infrared and optical masers," Phys. Rev., vol. 112, pp. 1940-1949, 1958.

[34] W. A. Edson, "Noise in oscillators," in Proc. Radio Eng., 1960, vol. 48, pp. 1454-1466.

[35] H. Rokhsari, M. Hossein-Zadeh, A. Hajimiri, and K. J. Vahala, "Brownian noise in radiation-pressure-driven micromechanical oscillators," Appl. Phys. Lett., vol. 89, no. 6, pp. 261109-1-261109-3, 2006.
[36] K. J. Vahala, "Back-action limit of linewidth in an optomechanical oscillator," Phys. Rev. A., vol. 78, p. 023832, 2008.

[37] G. Anetsberger, R. Rivière, A. Schliesser, O. Arcizet, and T. J. Kippenberg, "Ultralow-dissipation optomechanical resonators on a chip," Nature Photon., vol. 2, pp. 627-633, 2008.

[38] F. R. Blom, S. Bouwstra, M. Elwenspoek, and J. H. J. Fluitman, "Dependence of the quality factor of micromachined silicon beam resonators on pressure and geometry," J. Vac. Sci. Technol. B, vol. 10, no. 1, pp. 19-26, 1992.

[39] M. Hossein-Zadeh and K. J. Vahala, "Observation of injection locking in an optomechanical RF oscillator," Appl. Phys. Lett., vol. 93, pp. 1911151-191115-3, 2008.

[40] X. S. Yao and L. Maleki, "Optoelectronic microwave oscillator," J. Opt Soc. Amer. B, vol. 13, no. 8, pp. 1725-1735, 1996.

[41] M. Hossein-Zadeh and A. F. J. Levi, " $14.6 \mathrm{GHz} \mathrm{LiNbO}_{3}$ microdisk photonic self-homodyne RF receiver," IEEE Trans. Microw. Theory Tech. vol. 54, no. 2, pp. 821-831, Feb. 2006.

[42] M. Hossein-Zadeh and A. F. J. Levi, "Self-homodyne photonic microwave receiver architecture based on optical modulation and filtering," Microw. Opt. Technol. Lett., vol. 50, no. 2, pp. 345-350, 2008.

[43] M. Li, W. H. P. Pernice, C. Xiong, T. Baehr-Jones, M. Hochberg, and H. X. Tang, "Harnessing optical forces in integrated photonic circuits," Nature, vol. 456, pp. 458-458, 2008.

[44] M. Eichenfield, R. Camacho, J. Chan, K. J. Vahala, and O. Painter, "A picogram- and nanometre-scale photonic-crystal optomechanical cavity," Nature, vol. 459, pp. 550-556, 2009.

[45] T. J. Kippenberg, J. Kalkman, A. Polman, and K. J. Vahala, "Demonstration of an erbium-doped microdisk laser on a silicon chip," Phys. Rev. A., vol. 74, pp. 051802(R)-1-051802(R)-4, 2006.

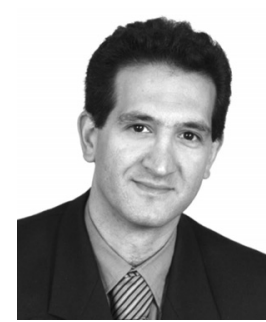

Mani Hossein-Zadeh (S'00-M'05) received the B.S. and M.S. degrees in physics from Sharif University of Technology, Tehran, Iran, in 1995 and 1997, respectively, and the M.S. and Ph.D. degrees in electrical engineering from the University of Southern California (USC), Los Angeles, in 2001 and 2004, respectively.

From 1995 to 1998, he was a Research Assistant in the Medical Physics Laboratory, Sharif University of Technology, where he was engaged in experimental work nonlinear optics and laser systems. From 1999 to 2005, he was a Research Assistant with the Advanced Network Technology Laboratory, USC, where he performed research on microwave photonics, specifically microdisk modulators and photonic RF receiver design. From 2005 to 2008, he was a Postdoctoral Scholar with Vahala Research Group and Center For Physics of Information, California Institute of Technology (Caltech), where he was involved in fluidic optical resonators, free microtoroid optical resonators, and optomechanical interaction in ultrahigh- $Q$ optical microresonators. He is currently an Assistant Professor of electrical and computer engineering at the University of New Mexico, Albuquerque.

Dr. Hossein-Zadeh is a member of the Optical Society of America and the Institute of Physics.

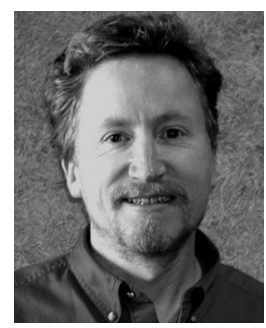

Kerry J. Vahala (S'82-M'84-SM'03) received the B.S. and Ph.D. degrees in applied physics, and the M.S. degree in electrical engineering from California Institute of Technology, Pasadena, in 1980, 1985, and 1981, respectively.

$\mathrm{He}$ is currently the Ted and Ginger Jenkins Professor and a Professor of applied physics in the California Institute of Technology. He was engaged in quantum-well laser dynamics that led to the widespread deployment of semiconductor lasers in optical communications. He has also pioneered microresonator devices operating in the $Q$-regime above 100 million. His current research interests include a range of optomechanical phenomena associated with radiation pressure in these devices.

Prof. Vahala was the Program Co-Chair for the Conference on Lasers and Electro-Optics (CLEO 1999) and the General Chair for CLEO 2001. He received the IEEE Sarnoff Award for his research on quantum well lasers, was the first recipient of the Richard P. Feynman Fellowship, received an Alexander Von Humboldt Research Award, and is a Fellow of the Optical Society of America. $\mathrm{He}$ also has received the Presidential Young Investigator and the Office of Naval Research Young Investigator Awards. 